\title{
Impact of colistin plasma levels on the clinical outcome of patients with infections caused by extremely drug-resistant Pseudomonas aeruginosa
}

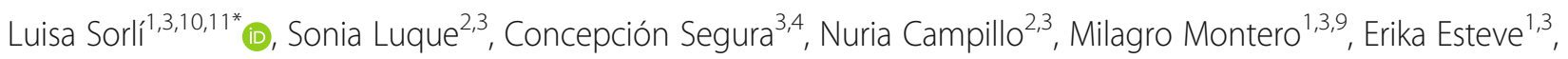
Sabina Herrera ${ }^{1,3}$, Natividad Benito ${ }^{5,6,9}$, Francisco Alvarez-Lerma ${ }^{7,3,9}$, Santiago Grau ${ }^{2,3,9}$

and Juan Pablo Horcajada 1,3,8,10,1 **

\begin{abstract}
Background: Colistin has a narrow therapeutic window with nephrotoxicity being the major dose-limiting adverse effect. Currently, the optimal doses and therapeutic plasma levels are unknown.

Methods: Prospective observational cohort study, including patients infected by colistin-susceptible $P$. aeruginosa treated with intravenous colistimethate sodium (CMS). Clinical data and colistin plasma levels at steady-state $\left(C_{s s}\right)$ were recorded. The primary and secondary end points were clinical cure and 30-day all-cause mortality.

Results: Ninety-one patients were included. Clinical cure was observed in 72 (79\%) patients. The mean (SD) $C_{\text {ss }}$ was $1.49(1.4) \mathrm{mg} / \mathrm{L}$ and $2.42(1.5) \mathrm{mg} / \mathrm{L}(p=0.01)$ in patients who achieved clinical cure and those who not, respectively. Independent risk factors for clinical failure were male sex (OR 5.88; 95\% Cl 1.09-31.63), APACHE II score (OR 1.15; 95\% Cl 1.03-1.27) and nephrotoxicity at the EOT (OR 9.13; 95\% Cl 95\% 2.06-40.5). The 30-day mortality rate was 30.8\%. Risk factors for 30-day mortality included the APACHE II score (OR 1.98; $95 \% \mathrm{Cl} 1-1.20)$, the McCabe score (OR 2.49; $95 \% \mathrm{Cl} 1.14-5.43$ ) and the presence of nephrotoxicity at the end of treatment (EOT) (OR 3.8; 95\% Cl 1.26-11.47).
\end{abstract}

Conclusion: In this series of patients with infections caused by XDR $P$. aeruginosa infections, $C_{s s}$ is not observed to be related to clinical outcome.

Keywords: Colistin, Mortality, Plasma concentration, Pseudomonas aeruginosa, Extremely drug-resistant, Nephrotoxicity

\section{Background}

The increasing incidence of infections due to multidrugresistant (MDR) gram-negative bacteria (GNB) is a concern worldwide due to high morbidity and mortality [1] and the lack of effective antimicrobials in the drugdevelopment pipeline [2]. Of particular concern are MDR Pseudomonas aeruginosa, Acinetobacter baumannii and Klebsiella pneumoniae [3]. For infections with these organisms, the only therapeutic option may be colistin,

\footnotetext{
*Correspondence: Isorli@parcdesalutmar.cat; 94021@hospitaldelmar.cat; jhorcajada@parcdesalutmar.cat

${ }^{1}$ Infectious Disease Service, Hospital del Mar, Barcelona, Spain

Full list of author information is available at the end of the article
}

which was discovered in the 1940s but never subjected to contemporary drug-development procedures. Colistin exhibits concentration-dependent bacterial killing, and its therapeutic efficacy depends on the ratio of the peak level to the minimal inhibitory concentration (MIC) or the area under the curve (AUC) to the MIC [4-6]. Colistin has a narrow therapeutic window, with nephrotoxicity being the most important dose-limiting adverse effect $[4,7]$. Some non-comparative studies have reported that colistin has an acceptable efficacy and safety profile in the treatment of infections caused by MDRGNB [8-10]. However, lower success rates are shown when compared with alternatives [11]. Although some 
clinical factors have been proposed as possible causes of this lower success rate, one important issue in this scenario is the shortage of available pharmacokinetic and pharmacodynamic data to guide the proper use of colistin in daily clinical practice [12].

Some recent studies have demonstrated that the current colistimethate sodium (CMS) dosage regimens are associated with suboptimal colistin concentrations and suboptimal pharmacokinetic targets for many strains of GN$\mathrm{GNB}$, and limited information is available regarding the optimal doses and the levels of colistin in plasma that provides a therapeutic effect. In addition, to date, no clinical studies have assessed the possible relationship between plasma colistin concentrations and clinical outcome. The aim of this study was to assess the possible relationship between colistin plasma concentration $\left(\mathrm{C}_{\mathrm{ss}}\right)$ and clinical cure and 30-day all-cause mortality in patients with infections due to colistin-susceptible extremely drug-resistant (XDR) $P$. aeruginosa who had been treated with intravenous CMS. The presence of nephrotoxicity at the end of treatment (EOT) was also analyzed.

\section{Methods}

\section{Study population and data collection}

A prospective observational cohort study was conducted between January 2009 and January 2013 at a 450-bed teaching hospital in Barcelona, Spain. All patients who had microbiologically documented infections due to colistin-susceptible XDR-P.aeruginosa and were administered CMS for at least $72 \mathrm{~h}$, were included in the study. Only microbiologically documented and clinically defined infections caused by colistin-susceptible XDR $P$. aeruginosa treated with colistin were considered. Acute bronchitis and tracheitis were excluded from the analysis due to the difficulty in differentiating true infection from colonization in patients with chronic obstructive pulmonary disease (COPD).

Patients were identified through the hospital's pharmacy registry of antibiotic use. Patients were excluded if they were $<18$ years old, pregnant, breastfeeding, on renal replacement therapy prior CMS treatment or if they had received CMS treatment for less than $72 \mathrm{~h}$. Patients who after a cured infection episode received another CMS curse due to another infection by XDR-P. aeruginosa, were considered as 2 different cases.

Patients were followed until hospital discharge or death.

The ethical committee of the hospital (Comité Ètic de Investigació Clínica del Parc de Salut Mar) approved the study. Informed consent was obtained form all participating patients or their legal representatives.

A standardized form was used to record patient characteristics, including age, sex, body weight, body mass index, data on the indication for CMS, the CMS administration schedule, the daily CMS dose in millions of international units (IU), the cumulative CMS dose (in millions of IU), the duration of treatment and the colistin plasma concentration at steady state $\left(\mathrm{C}_{\mathrm{ss}}\right)$. Additional clinical data collected for the study included the following: baseline glomerular filtration rate (GFR), calculated using the abbreviated Modification of Diet in Renal Disease equation (MDRD) [13]; the presence of chronic kidney disease (CKD) at baseline, which was diagnosed when the GFR was $<60 \mathrm{~mL} / \mathrm{min} / 1.73 \mathrm{~m}^{2}$ for $\geq 3$ months; the presence of underlying comorbidities, evaluated by the Charlson comorbidity index [14]; the severity of disease at the time of the first CMS dose, stratified according to the Acute Physiology and Chronic Health Evaluation (APACHE II) [15]; concomitant nephrotoxic drugs (aminoglycosides, vancomycin, nonsteroidal antiinflammatory drugs, intravenous radiocontrast agent, loop diuretic, angiotensin-converting-enzyme inhibitors, angiotension II receptor antagonist and ciclosporine); the causative organism and in vitro susceptibility data; the use of combined antibiotic treatment; and the clinical response to therapy. Data were prospectively collected for 30 days from the onset of CMS treatment, unless otherwise specified.

The primary end point was clinical cure, and the secondary 30-day all-cause mortality.

\section{Definitions and microbiological testing}

Infections were defined according to the Centers for Disease Control and Prevention (CDC) [16]. The patient's clinical status at the beginning of CMS treatment was defined as infection, severe sepsis or septic shock, According to standard definitions [17], severe sepsis was considered when an acute organ dysfunction secondary to documented or suspected infection and septic shock was defined as having a severe sepsis plus hypotension not reversed with fluid resuscitation.

Clinical cure and failure were defined as resolution and persistence/worsening, respectively, of symptoms and signs of infection.

Thirty-day all-cause mortality was considered as death during the hospitalization.

XDR-Pseudomonas aeruginosa was defined according to the $\mathrm{CDC} / \mathrm{ECDC}$ criteria [18] as an isolate non-susceptible to at least 1 agent in all but 2 or fewer categories: aminoglycosides, antipseudomonal carbapenems, antipseudomonal cephalosporins, antipseudomonal fluoroquinolones, antipseudomonal penicillins + $\beta$-lactamase inhibitor, monobactams, phosphonic acids and polymyxin.

Combined antibiotic treatment consisted of CMS plus ceftazidime or carbapenem in high-dose extended infusion, or CMS plus amikacin.

Routine identification and susceptibility testing of causative microorganisms were first performed by microdilution using the gram-negative (GN) breakpoint panel for 
non-fermenting GNB of the MicroScan ${ }^{\circ}$ WalkAway system (Siemens Diagnostic Inc., CA). The colistin Minimal Inhibitory Concentration (MIC) was determined by microdilution using cation-adjusted $\mathrm{MHB}$; the isolates were considered susceptible if the MIC value was $<2 \mathrm{mg} / \mathrm{L}$ according to the Clinical and Laboratory Standards Institute (CLSI). Performance standards for antimicrobial susceptibility testing; informational supplement. CLSI document M100-SCLSI, Wayne, PA (2013).

\section{CMS administration and colistin plasma level measurements}

CMS for injection was used and dosed in millions of IU throughout the study. The drug was administered intravenously in $100 \mathrm{~mL}$ of normal saline over $30 \mathrm{~min}$ in the commercially available colistimethate formulation for injection (GES GenéricosEspañoles ${ }^{\oplus}$ ), with each vial containing $80 \mathrm{mg}$ CMS (equivalent to 1 million IU of CMS and about $30 \mathrm{mg}$ of colistin base activity). As the policy of our hospital, CMS is reserved for the treatment of infections due to bacteria that are resistant to carbapenems and other antipseudomonal antibiotics except colistin and, eventually, aminoglycosides. Dose selection was at the discretion of the responsible clinicians. CMS doses ranged from 1 to 3 million IU every $8 \mathrm{~h}$ (3-9 millions IU daily). The following dose adjustments were made according to the package insert's recommendations in patients with impaired renal function: GFR $\geq 76 \mathrm{~mL} / \mathrm{min} /$ $1.73 \mathrm{~m}^{2}, 4-6$ million IU daily in three doses; GFR 40 to $75 \mathrm{~mL} / \mathrm{min} / 1.73 \mathrm{~m}^{2}, 2-3$ million IU daily in 2 doses; GFR $25-40 \mathrm{~mL} / \mathrm{min} / 1.73 \mathrm{~m}^{2}, 1.5-2$ million IU daily divided into 1 or 2 doses; and GFR $<25 \mathrm{~mL} / \mathrm{min} / 1.73 \mathrm{~m}^{2}$, 0.6-1 million IU daily every $36 \mathrm{~h}$. No loading-dose was administered to any included patient.

Measurement of the plasma colistin concentration was performed at the fourth day of treatment, when was assumed that the colistin concentrations had reached the steady state. The colistin plasma trough concentrations $\left(\mathrm{C}_{\text {min }}\right)$ were measured just before the administration of CMS. The maximum plasma concentrations $\left(C_{\max }\right)$ were obtained $30 \mathrm{~min}$ after the end of the CMS infusion ( $1 \mathrm{~h}$ after the start of infusion). A specialized nurse performed extraction of blood. Samples were immediately placed in a portable refrigerator $\left(4^{\circ} \mathrm{C}\right)$ to be processed in a refrigerated centrifuge $\left(4{ }^{\circ} \mathrm{C}\right)$ at the laboratory. Colistin concentrations in the plasma were determined using a validated high-performance liquid chromatography (HPLC) method as reportedby Li et al. [19], with minor modifications as described previously by our group [20].

Because the $C_{\max }(1.66 \pm 1.40 \mathrm{mg} / \mathrm{L})$ and $C_{\min }(1.71 \pm$ $1.42 \mathrm{mg} / \mathrm{L})$ were highly correlated $(R=0.98 ; P<0.001)$, only one of these variables was used throughout the study as a marker of colistin plasma exposure. Following
Couet et al. [21], $\mathrm{C}_{\min }$ was chosen because it is more convenient from a practical view point and because due to the colistin pharmacokinetic behavior, there can be CMS hydrolysis to colistinafter sample extraction resulting in an overestimation of the real in vivo colistin plasma concentration. This phenomenon can be minimized when CMS plasma concentrations are lower what occurs at the end of the dosing interval (when the $\mathrm{C}_{\text {min }}$ is measured). Throughout the present paper, we have used the term "average colistin concentration at steady state $\left(\mathrm{C}_{\mathrm{ss}}\right)$ " to refer to the $\mathrm{C}_{\mathrm{min}}$.

\section{Efficacy and nephrotoxicity assessment}

Thirty-day mortality was defined as death occurring within 30 days of beginning CMS treatment.

Clinical cure was defined as having resolved clinical signs and symptoms and/or no need for additional antibiotic therapy. Clinical failure was defined as an inadequate response to antibiotic therapy, with worsening, new/recurrent signs and symptoms or death.

The serum creatinine level and estimated glomerular filtration rate (GFR) were recorded at baseline, at the moment of colistin plasma extraction (day 4), on day 7 and at the EOT. We chose these time points because in our center, blood tests are performed routinely in all inpatients at least once a week and at the end of treatment. The RIFLE criteria (Table 1) estimated with exclusion of the urinary output criterion, were used for the detection and stratification of acute kidney injury (AKI) [22]. AKI during CMS treatment was defined as a 1.5fold or more increase in serum creatinine and/or a decrease in the GFR of $25 \%$ or more. These criteria needed to be fulfilled for at least 2 consecutive measurements $24 \mathrm{~h}$ apart during CMS treatment.

\section{Statistical analysis}

Dichotomous data were compared using a $x^{2}$ or Fisher's exact test. Normally distributed continuous data are expressed as the means with standard deviations (SD) and were compared using the $t$-test. Otherwise, values are

Table 1 Definition of the RIFLE criteria to assess renal injury

\begin{tabular}{ll}
\hline Category criteria & Definition \\
\hline Risk (R) & Increased creatinine level $\times 1.5$ or ${ }^{1}$ \\
& GFR decrease $>25 \%$ \\
Injury (I) & Increased creatinine level $\times 2$ or ${ }^{1}$ \\
& GFR decrease $>50 \%$ \\
Failure (F) & Increased creatinine level $\times 3{ }^{1}$ \\
& GFR decrease $>75 \%$, or creatinine \\
& level $>4$ mg/dL \\
& Persistent acute renal failure or \\
Loss (L) & loss of function for $>4$ weeks \\
& ESKD for $>3$ months \\
\hline
\end{tabular}

${ }^{1}$ GFR glomerular filtration rate 
presented as the means with interquartile range (IQR) and were compared using the Mann-Whitney $U$-test.

Logistic regression was used to explore risk factors associated with clinical cure and 30-day all- cause mortality.

Univariate analyses were performed separately for each of the risk factor variables to ascertain the odds ratios (ORs) and 95\% confidence intervals (CI). For continuous variables found significant in the analysis, the threshold breakpoint was determined $\left(\mathrm{C}_{\mathrm{ss}}\right)$. All clinically important covariates and those with a $P$ value $<0.2$ in the univariate analyses were included in the multivariate analysis.

$\mathrm{C}_{\mathrm{ss}}$ was also analyzed and included in the model as a dichotomous variable by defining one set point. According to Garonzik et al. [6], this set point was $1.25 \mathrm{mg} / \mathrm{L}$, corresponding to a target $\mathrm{AUC}_{0-24}$ of $60 \mathrm{mg} . \mathrm{h} / \mathrm{L}$ for a P. aeruginosa strain with an MIC value of $0.5 \mathrm{mg} / \mathrm{L}$, which is the predominant strain at our center.

A backward selection process was utilized in which the results of the Wald test for individual parameters were examined. The least significant effect that did not meet the level for staying in the model was removed and it remained excluded.

Multivariate logistic regression models were assessed using the Hosmer-Lemeshow goodness-of-fit test. The discriminatory power of the predictive model was established by means of receiver operating characteristic (ROC) curves. For all analyses, a two-sided $P$ value $<0.05$ was considered to be statistically significant. The Statistical Package for the Social Sciences (SPSS, version 15.0) was used for the statistical analysis.

\section{Results}

During the study period, 124 patients with colistinsusceptible XDR $P$. aeruginosa infections treated with intravenous CMS were evaluated. Of these, 33 (26.6\%) were diagnosed with acute bronchitis or tracheitis and were excluded. Thus, 91 patients were finally included in the analysis. Three patients developed 2 infectious episodes due to XDR-P. aeruginosa (patient 1: two episodes of UTI in 3 months. The second episode was considered a new infection; patient 2: two episodes of UTI in 5 months. The second episode was considered a new infection; patient 3: one episode of ventriculitis and one UTI), and each infection was included as a separate case. The clinical and demographic characteristics of the included patients are shown in Table 2 .

Clinical cure was observed in 72 (79.1\%) patients. Table 3 shows the clinical characteristics of patients with clinical cure and clinical failure. The patients with clinical failure were mainly men with higher APACHE II indices. Additionally, they had achieved higher $\mathrm{C}_{\mathrm{ss}}$ values and were more likely to have developed AKI than patients with clinical cure. In the multivariate analysis, factors related to clinical failure were male sex (OR 5.88; 95\% CI 1.09-31.63, $P=0.039$ ), APACHE II score (OR 1.15 ; $95 \%$ CI $1.03-1.27, P=0.013)$ and the presence of AKI at the EOT (OR 9.13; 95\% CI 95\% 2.06-40.5, $P=0.004$ ).

Twenty eight patients died during hospitalization so the 30 -day all-cause mortality rate was $30.8 \%$. However, only 13 patients died due to $P$. aeruginosa infection leading to a infection-related mortality rate of $14.3 \%$. These data reveals that 15 out of the 28 patients who died (53.6\%) did so for reasons other than the infection itself. Causes of death in these patients were mainly related to their comorbid conditions (4 patients of COPD complications, 2 of cirrhosis, 2 of heart failure, 1 because malignancy, 2 of hematological malignancies, 1 because complications of Clostridium difficile infection) or postsurgical complications (3 patients). So only one death in this group could be related to colistin treatment and it was one patient who died because a Clostridium difficile infection. However, this patient has also been treated with other antibiotics in addition to colistin. The patients who died were mainly men with higher Charlson, APACHE II and McCabe scores, higher $\mathrm{C}_{\mathrm{ss}}$ values and a higher likeliness of having developed AKI during treatment (Table 4). In the multivariate analysis, the following independent risk factors for 30-day mortality were identified: APACHE II score (OR 1.98; 95\% CI 1-1.20, $P=0.046$ ), McCabe score (OR 2.49; 95\% CI 1.14-5.43, $P=0.021$ ), and the presence of AKIat the EOT (OR 3.8; 95\% CI 1.26-11.47, $P=0.018$ ).

Twelve patients (13.2\%) presented AKI prior CMS treatment. This factor was not related with 30-day allcause mortality $(P=0,5)$ or clinical cure $(P=1)$. Impairment of renal function during CMS treatment was observed in $30(33 \%)$ patients on day 7 and in $49(53.8 \%)$ at the EOT. The distribution of AKI on the basis of the RIFLE criteria was 19 (20.9\%) R, 9 (9.9\%) I and 2 (2.2\%) F on day 7 of treatment; and 12 (13.2\%) R, 27 (29.7\%) I and $10(11 \%) \mathrm{F}$ at the EOT. The CMS dose was modified because of AKI in $16(17.5 \%)$ patients. At the end of follow-up, the GFR returned to baseline in 32 (35.1\%) patients. Two patients $(2.1 \%)$ persisted with some grade of impairment of renal function, 15 (16.4\%) died and 3 (3.2\%) patients had no follow-up information regarding renal function.

\section{Discussion}

Currently, there is a lack of pharmacokinetic and pharmacodynamic data to guide the proper use of colistin in daily clinical practice. We have observed that $\mathrm{C}_{\mathrm{ss}}$ is not related to clinical cure and 30-day all-cause mortality in a prospective series of patients infected with colistinsusceptible XDR $P$. aeruginosa. 
Table 2 Clinical and demographic characteristics of included patients

\begin{tabular}{|c|c|}
\hline & Included patients $(n=91)$ \\
\hline Age, years* & $67(24-88)$ \\
\hline Male sex, $n(\%)$ & $66(72.5)$ \\
\hline APACHE $\|^{*}$ & $11(2-28)$ \\
\hline \multicolumn{2}{|l|}{ Co-morbidities, $n$ (\%): } \\
\hline Malignancy & $14(15.4)$ \\
\hline Cardiovascular & $26(28.6)$ \\
\hline Pulmonary & $33(36.3)$ \\
\hline Diabetes Mellitus & $21(23.1)$ \\
\hline Urogenital $^{\mathrm{a}}$ & $14(15.4)$ \\
\hline Hepatic & $8(8.8)$ \\
\hline Haematological ${ }^{\mathrm{b}}$ & $12(13.2)$ \\
\hline Neurological ${ }^{c}$ & $18(19.2)$ \\
\hline Charlson score* & $4(0-10)$ \\
\hline McCabe score** & $1.48 \pm 0.64$ \\
\hline Patients with $\mathrm{CKD}^{1}$ at baseline & 19 (20.9) \\
\hline \multicolumn{2}{|l|}{ Type of infection, $n(\%)$ : } \\
\hline Pneumonia & $24(24.6)$ \\
\hline Urinary tract infection & $22(24.2)$ \\
\hline Skin and soft tissue infection & $11(12.1)$ \\
\hline Organ space surgical site infection & $10(11)$ \\
\hline Bacteremia & $6(6.6)$ \\
\hline Other & $18(19.8)$ \\
\hline Hospital-acquired infection, $n(\%)$ & $86(94.5)$ \\
\hline \multicolumn{2}{|l|}{ Department of hospitalization: } \\
\hline Medical & $44(48.2)$ \\
\hline Surgical & $32(35.2)$ \\
\hline $\mathrm{ICU}^{2}$ & $15(16.2)$ \\
\hline \multicolumn{2}{|l|}{ Admission diagnosis category: } \\
\hline Infection & $26(28.6)$ \\
\hline Other-medical & $35(38.5)$ \\
\hline Other-surgical & $30(33)$ \\
\hline $\mathrm{CMS}^{3}$ daily dose (millions of $\left.\mathrm{IU}^{4}\right)^{* *}$ & $5.45 \pm 2.21$ \\
\hline $\mathrm{CMS}^{3}$ total dose (millions of $\left.\mathrm{IU}^{4}\right)^{* *}$ & $108.36 \pm 96.41$ \\
\hline $\mathrm{CMS}^{3}$ duration of treatment, days** & $20.18 \pm 16.01$ \\
\hline Inhaled $\mathrm{CMS}^{3}, n(\%)$ & $14(15.4)$ \\
\hline Combined antimicrobial therapy, $n$ (\%) & $46(50.5)$ \\
\hline$C_{s s}^{5}(m g / L)^{* *}$ & $1.67 \pm 1.42$ \\
\hline $\mathrm{C}_{\mathrm{ss}}^{5}>1.28(\mathrm{mg} / \mathrm{L}), n(\%)$ & $46(50.5)$ \\
\hline $\mathrm{C}_{S S}^{5} / \mathrm{MIC}^{6 * *}$ & $3.43 \pm 2.91$ \\
\hline $\mathrm{AKI}^{7}$ prior to $\mathrm{CMS}^{3}$ treatment, $n(\%)$ & $12(13.2)$ \\
\hline
\end{tabular}

Table 2 Clinical and demographic characteristics of included patients (Continued)

\begin{tabular}{ll}
\hline Patients with AKI at day $7, n(\%)$ & $19(20.9)$ \\
R (Risk) & $9(9.9)$ \\
I (Injury) & $2(2.2)$ \\
F (Failure) & \\
Patients with AKI ${ }^{7}$ at the EOT $^{8}, n(\%)$ & $12(13.2)$ \\
R (Risk) & $27(27.7)$ \\
I (Injury) & $10(11)$ \\
F (Failure) & $72(79.1)$ \\
Clinical response, $n$ (\%) & $28(30)$ \\
30-Day all-cause mortality, $n$ (\%) & $67 \pm 53.97$ \\
Hospital length-of-stay (days) &
\end{tabular}

${ }^{1} \mathrm{CKD}$ chronic kidney disease, ${ }^{2} \mathrm{ICU}$ intensive cure unit, ${ }^{3} \mathrm{CMS}$ colistin

methanesulphonate, ${ }^{4} / U$ international units, ${ }^{5} C_{s s}$ colistin plasma concentration

at steady-state, ${ }^{6} \mathrm{MIC}$ minimal inhibitory concentration, ${ }^{7} \mathrm{AKI}$ acute kidney injury,

${ }^{8} \mathrm{EOT}$ end of treatment

*median (range)

**mean \pm SD

${ }^{a}$ Among urogenital co-morbidities were, renal disease, kidney stones and

obstructive uropathy

${ }^{\mathrm{b}}$ Among haematological co-morbidities were haemopoietic and lymphoreticular malignances

'Among neurological co-morbidities were Alzheimer's disease, stroke, miastenia gravis, sclerosis and any kind of dementia

Clinical cure in this series of patients was $79.1 \%$ and was similar to those reported in previous studies [9, 10, 23-25]. Factors related with clinical failure were APACHE II score and the presence of nephrotoxicity at the EOT. We highlight the fact that colistin plasma levels were not related with clinical cure. This finding is in some disagreement with recent studies suggesting that higher doses of CMS might be more appropriate for the treatment of infections caused by MDR-GNB [6, 26, 27]. However, some recent clinical experiences did not observe a significant association between the CMS dose and the clinical or microbiological outcomes. Yilmaz et al. studied daily doses of 3 and 6 million IU CMS in the treatment of infections caused by MDR-GNB and observed no differences in the end points [28]. In another study, Zaidi et al. reported that low CMS doses could also be an effective option in the treatment of infections caused by MDR-GNB [29]. The reasons for this apparent lack of relationship between colistin concentrations or doses, and clinical outcome need to be studied in depth in future studies.

In terms of PK analysis, data from the present study are consistent with the results of these previous studies, in the sense that the classic CMS dosage regimens are associated with suboptimal colistin concentrations and suboptimal pharmacokinetic targets for many strains of GN-GNB. However, based on the population model described by Garonzik et al. [6], we defined a set point corresponding to a target $\mathrm{AUC}_{0-24}$ of $60 \mathrm{mg} . \mathrm{h} / \mathrm{L}$ for a 
Table 3 Clinical and demographic characteristics of patients with and without clinical cure

\begin{tabular}{|c|c|c|c|}
\hline & $\begin{array}{l}\text { Clinical cure } \\
(n=72)\end{array}$ & $\begin{array}{l}\text { Clinical failure } \\
(n=19)\end{array}$ & $P$-value \\
\hline Male sex & $49(68)$ & $17(89.5)$ & 0.06 \\
\hline Age, years* & $66.5(24-88)$ & $67(41-84)$ & 0.59 \\
\hline APACHE $\|^{*}$ & $11(2-28)$ & $13.5(6-24)$ & 0.05 \\
\hline \multicolumn{4}{|l|}{ Co-morbidities, n (\%): } \\
\hline Malignancy & $11(15.3)$ & $3(15.8)$ & 1 \\
\hline Cardiovascular & $21(29.2)$ & $5(26.3)$ & 1 \\
\hline Pulmonary & $24(33.3)$ & $9(47,4)$ & 0,26 \\
\hline Diabetes Mellitus & $20(27.8)$ & $1(5.3)$ & 0.06 \\
\hline Urogenital $^{\mathrm{a}}$ & $11(15.3)$ & $3(15.8)$ & 1 \\
\hline Hepatic & $8(11.1)$ & $8(0)$ & 0.2 \\
\hline Haematological $^{b}$ & $9(12.5)$ & $3(15.8)$ & 0.7 \\
\hline Neurological $^{c}$ & $16(22.2)$ & $2(10.5)$ & 0.34 \\
\hline Charlson* & $4.5(0-10)$ & $4(1-9)$ & 0.73 \\
\hline McCabe ${ }^{* *}$ & $1.4 \pm 0.6$ & $1.7 \pm 0.7$ & 0.11 \\
\hline Severe sepsis, $n$ (\%) & $49(68.1)$ & $9(47.4)$ & 0.095 \\
\hline Shock & $6(8.3)$ & $1(5.3)$ & 0.65 \\
\hline Patients with CKD at baseline & $16(22.2)$ & $3(15.8)$ & 0.53 \\
\hline \multicolumn{4}{|l|}{ Department of hospitalization: } \\
\hline Medical & $38(52.8)$ & $6(31.6)$ & 0.16 \\
\hline Surgical & $23(32)$ & $9(47.4)$ & \\
\hline$I C U^{2}$ & $11(15.3)$ & $4(21.1)$ & \\
\hline \multicolumn{4}{|l|}{ Admission diagnosis category: } \\
\hline Infection & $19(26.4)$ & $7(36.8)$ & \\
\hline Other-medical & $30(41.7)$ & $5(26.3)$ & \\
\hline Other-surgical & $23(3.9)$ & $7(36.8)$ & 0.45 \\
\hline CMS daily dose (millions IU)** & $5.3 \pm 2.3$ & $6.2 \pm 2.1$ & 0.094 \\
\hline CMS total dose (millions IU) ${ }^{* *}$ & $105.91 \pm 88.9$ & $141.2 \pm 129.5$ & 0.45 \\
\hline CMS duration of treatment, days** & $20.66 \pm 16.1$ & $22.6 \pm 18.5$ & 0.81 \\
\hline Combined treatment, $n$ (\%) & 35 (48.6) & $11(57.9)$ & 0.47 \\
\hline $\mathrm{C}_{\mathrm{ss}}(\mathrm{mg} / \mathrm{mL})^{* *}$ & $1.49 \pm 1.4$ & $2.42 \pm 1.49$ & 0.01 \\
\hline $\mathrm{C}_{\mathrm{ss}}>1.25(\mathrm{mg} / \mathrm{mL}), n(\%)$ & $32(45.1)$ & $14(73.7)$ & 0.027 \\
\hline $\mathrm{C}_{5 s} / \mathrm{MIC}^{* *}$ & $3.13 \pm 2.9$ & $4.61 \pm 2.86$ & 0.03 \\
\hline AKI at day $7, n(\%)$ & $18(25)$ & $12(63.2)$ & 0.002 \\
\hline AKI at the EOT, $n(\%)$ & $33(45.8)$ & $16(84.2)$ & 0.003 \\
\hline Length of stay, days** & $69.16 \pm 59.5$ & $70.8 \pm 39.1$ & 0.19 \\
\hline
\end{tabular}

${ }^{1} \mathrm{CKD}$ chronic kidney disease, ${ }^{2} \mathrm{ICU}$ intensive cure unit, ${ }^{3} \mathrm{CMS}$ colistin methanesulphonate, ${ }^{4} I U$ international units, ${ }^{5} C_{s s}$ colistin plasma concentration at steady-state, ${ }^{6} \mathrm{MIC}$ minimal inhibitory concentration, ${ }^{7} \mathrm{AKI}$ acute kidney injury, ${ }^{8} \mathrm{EOT}$ end of treatment

*median (range)

**mean \pm SD

${ }^{a}$ Among urogenital co-morbidities were, renal disease, kidney stones and obstructive uropathy

${ }^{\mathrm{b}}$ Among haematological co-morbidities were haemopoietic and

lymphoreticular malignances

'Among neurological co-morbidities were Alzheimer's disease, stroke, miastenia gravis, sclerosis and any kind of dementia
Table 4 Clinical and demographic characteristics of patients who died and those who survived

\begin{tabular}{|c|c|c|c|}
\hline & $\begin{array}{l}\text { Died } \\
(n=28)\end{array}$ & $\begin{array}{l}\text { Survived } \\
(n=63)\end{array}$ & $P$-value \\
\hline Male sex & $24(85.7)$ & $42(66.7)$ & 0.06 \\
\hline Age, years* & $66.85(41-84)$ & $65.5(24-87)$ & 0.12 \\
\hline APACHE $\|^{*}$ & $14(5-27)$ & $10.5(2-28)$ & 0.047 \\
\hline \multicolumn{4}{|l|}{ Co-morbidities, $n$ (\%): } \\
\hline Malignancy & $6(21.4)$ & $8(12.7)$ & 0.35 \\
\hline Cardiovascular & $7(25)$ & $19(30.2)$ & 0.8 \\
\hline Pulmonary & $15(53.6)$ & $18(28.6)$ & 0.03 \\
\hline Diabetes Mellitus & $3(10.7)$ & 18 (28.6) & 0.1 \\
\hline Urogenital $^{a}$ & $6(21.4)$ & $8(12.7)$ & 0.35 \\
\hline Hepatic & $4(14.3)$ & $4(6.3)$ & 0.24 \\
\hline Haematological $^{\mathrm{b}}$ & $4(14.3)$ & $8(12.7)$ & 1 \\
\hline Neurological ${ }^{c}$ & $2(7.1)$ & $16(23.4)$ & 0.05 \\
\hline Charlson* & $5(1-9)$ & $4(0-10)$ & 0.039 \\
\hline McCabe** & $1.8 \pm 0.7$ & $1.3 \pm 0.6$ & 0.008 \\
\hline
\end{tabular}

Clinical status, $n(\%)$

$\begin{array}{llll}\text { Severe sepsis } & 15(53.6) & 43(68.3) & 0.17 \\ \text { Shock } & 2(7.1) & 5(7.9) & 0.89 \\ \text { Patients with CKD at baseline } & 7(25) & 12(19) & 0.52\end{array}$

Department of hospitalization:

$\begin{array}{llll}\text { Medical } & 12(42.9) & 32(50.8) & \\ \text { Surgical } & 11(39.3) & 21(33.3) & 0.78 \\ \text { ICU } & 5(17.9) & 10(15.9) & \end{array}$

Admission diagnosis category:

\begin{tabular}{|c|c|c|c|}
\hline Infection & $7(25)$ & $19(30.2)$ & \\
\hline Other-medical & $12(42.9)$ & $23(36.5)$ & 0.82 \\
\hline Other-surgical & $9(32.1)$ & $21(33.3)$ & \\
\hline MS daily dose (millions IU)** & $5.5 \pm 2.4$ & $5.4 \pm 2.2$ & 0.793 \\
\hline MS total dose (millions IU)** & $114.4 \pm 116.5$ & $113.3 \pm 91.5$ & 0.68 \\
\hline MS duration of treatment, days** & $20.3 \pm 16.5$ & $21.4 \pm 16.6$ & 0.88 \\
\hline Combined treatment, $n(\%)$ & $15(53.6)$ & $31(49.2)$ & 0.7 \\
\hline$-s s(m g / L)^{* *}$ & $2,1 \pm 1.4$ & $1.4 \pm 1,4$ & 0.011 \\
\hline$-s s>1.25(\mathrm{mg} / \mathrm{L}), n(\%)$ & $18(64.3)$ & $28(44.4)$ & 0.093 \\
\hline$=5 / M I C^{* *}$ & $4.2 \pm 2.7$ & $3.1 \pm 3$ & 0.048 \\
\hline $\mathrm{K} \mathrm{Kl}$ at day $7, n(\%)$ & $14(50)$ & $16(25.4)$ & 0.021 \\
\hline KKI at the EOT, $n(\%)$ & $20(71.4)$ & $29(46)$ & 0.025 \\
\hline ength of hospital stay, (days) ${ }^{* *}$ & $65.2 \pm 33.9$ & $71.5 \pm 63.2$ & 0.3 \\
\hline
\end{tabular}

${ }^{1} C K D$ chronic kidney disease, ${ }^{2} I C U$ intensive cure unit, ${ }^{3} C M S$ colistin methanesulphonate, ${ }^{4} I U$ international units, ${ }^{5} C_{s s}$ colistin plasma concentration at steady-state, ${ }^{6} \mathrm{MIC}$ minimal inhibitory concentration, ${ }^{7} \mathrm{AKI}$ acute kidney injury, ${ }^{8}$ EOT end of treatment

*median (range)

**mean \pm SD

${ }^{a}$ Among urogenital co-morbidities were, renal disease, kidney stones and obstructive uropathy

${ }^{\mathrm{b}} \mathrm{Among}$ haematological co-morbidities were haemopoietic and lymphoreticular malignances

'Among neurological co-morbidities were Alzheimer's disease, stroke, miastenia gravis, sclerosis and any kind of dementia 
P. aeruginosa with an MIC value of $0.5 \mathrm{mg} / \mathrm{L}$, and this set point was not related to the clinical outcomes or to 30-day all-cause mortality.

The 30-day all-cause mortality rate in our series was $30.9 \%$. Previous comparative and non-comparative studies have reported similar crude mortality rates $[8,11,30,31]$. The APACHE II score and the severity of underlying diseases have been previously reported as predictors of mortality in patients treated with CMS for infections caused by MDR-GNB [30]. In patients with ventilatorassociated pneumonia due to $P$. aeruginosa, the severity of the clinical presentation has been reported to be the main predictor of mortality [31]. In accordance with these studies, our series showed that the severity of the acute illness and of the underlying diseases were also independent risk factors for death.

An important finding of this study is that although $\mathrm{C}_{\mathrm{ss}}$ was not found to be a predictor of clinical failure or mortality in the multivariate model, it is interesting that $\mathrm{C}_{\mathrm{ss}}$ was statistically significant in the univariate model and that higher average $C_{s s}$ values were associated to clinical failure and also with 30-day all-cause mortality. In an attempt to address this finding, we analyzed the ratio of discontinuation or doses adjustment of colistin due to nephrotoxicity and there were no differences between groups. In our opinion this fact have to be studied in larger prospective studies but one possible hypothesis is that higher levels of colistin have been related to higher ratios of nephrotoxicity [20] and maybe nephrotoxicity and not colistin levels is the responsibly of this poor clinical outcome. In fact, the presence of AKI at the EOT was related to 30 -day all-cause mortality in a previous study carried by our group [20].

Of note, in our series the presence of nephrotoxicity at the EOT was associated with mortality and clinical failure. Information about the impact of nephrotoxicity during treatment with CMS on clinical outcomes is scarce. The data that have been reported are contradictory: although some studies have reported higher rates of mortality in patients with nephrotoxicity $[8,20]$, others have not found this association [32, 33]. Our findings suggest that the development of AKI during treatment with CMS seems to be a more important dose-limiting adverse event than previously recognized. According to our previously reported results showing rates of nephrotoxicity of $65-85 \%$ with colistin trough concentrations greater than $2.2 \mu \mathrm{g} / \mathrm{mL}$ [20], AKI during treatment with CMS could be controlled by monitoring colistin plasma concentrations.

We acknowledge some limitations of the current study. First is its observational design that makes difficult to draw stronger conclusions. Second, the sample was heterogeneous in terms of the infection source and the administered CMS dose. In this scenario, previous reports have demonstrated that patients with pneumonia responded less favorably to CMS therapy $[23,34]$ probably due to the poor drug penetration to the epithelial lining fluid. However this is a "real life" series with a significant number of difficult to treat infections that deserve to be studied in the antimicrobial resistance era. Another limitation is that we did not use the loading dose strategy because the protocol study was designed before this practice was recommended in the literature. Although recent published experiences have suggested that this strategy has not been validated with clinical data [32], without this loading dose it may be much longer to reach the steady-state. However colistin levels were extracted at the fourth day of treatment and therefore analyses have been done at the steady state. Fourth is the lack of specific analysis on the MICs of the isolated microorganism in each patient. The efficacy of antimicrobial therapy is not only based on drug exposure or pathogen susceptibility, instead it is an integrated function of both. The lack of this analysis is another possible source of confounding and should be evaluated in future studies. Aware of all these limitations and with the intention of overcoming some of these handicaps, our group has carried out a clinical trial (clinicaltrials.gov number NCT01845246) whose results are pending analysis and publication at this time.

Our findings highlight several issues. First, although it is currently accepted that there is a need for changes in the recommended CMS dosing regimens to achieve higher colistin plasma levels $[5,6]$, in our study, the $C_{\mathrm{ss}}$ was not related to a better clinical outcome. Second, our results suggest that the severity of the infection, the presence of comorbidities and the presence of AKI at the EOT are the most important factors related to the clinical outcome in XDR P. aeruginosa infections treated with intravenous CMS. Third, because of these findings, more prospective and homogeneous clinical studies should be conducted to evaluate the benefit of monitoring colistin concentrations in plasma in clinical practice. These studies have to assess the issue of the optimal dose of CMS in order to maximize clinical benefits and minimize toxicities.

\section{Conclusions}

In conclusion in this series of patients, colistin plasma levels were not observed to be related to clinical cure or mortality in patients with XDR $P$. aeruginosa infections treated with CMS. However these finding needs to be confirmed in future studies. Of note, the presence of AKI at the EOT is a risk factor for mortality and clinical failure. 


\section{Abbreviations}

AKI: Acute kidney injury; AUC: Area under the curve; CDC: Centers for Disease Control and Prevention; Cl: Confidence interval; CKD: Chronic kidney disease; $C_{\text {max }}$ : Maximum plasma concentrations; $C_{\min }$ : Minimum plasma concentrations; CMS: Colistimethate sodium; COPD: Chronic obstructive pulmonary disease; $C_{s s}$ : Colistin plasma levels at steady-state; EOT: End of treatment; GFR: Glomerular filtration rate; GNB: Gram negative bacteria; HPLC: High performance liquid chromatography; IQR: Interquartile range; IU: International units; MDR: Multidrug-resistant; MDRD: Modification of Diet in Renal Disease equation; MIC: Minimal inhibitory concentration; OR: Odds ratio; ROC curve: Receiving operating characteristic curve; SD: Standard deviation; XDR: Extremely drug-resistant

\section{Funding}

This work was supported by Fondo de Investigación Sanitaria (FIS) from Instituto de Salud Carlos III, Spanish Ministry of Health, Grant number PS09/01634 and from Spanish Ministry of Health and Social Policy, General Pharmacy Subdirection, Grant numbers EC10-165 and EC11-318. This study also received funding from European Regional Development Fund (FEDER: "A way of making Europe"). NB was funded by the Ministerio de Economía y Competitividad, Instituto de Salud Carlos III and cofinanced by the European Development Regional Fund "A way to achieve Europe", Spanish Network for the Research in Infectious Diseases (REIPI RD12/0015).

\section{Availability of data and materials}

All the data will be made available by the corresponding author upon request.

\section{Author's contributions}

Analyzed the data: LS, SL, JPH, SG, NB. Wrote paper: LS, SL. Designed the estudy: LS, SL, JPH, SG, NB. Conducted laboratory work: CS. Conducted pharmacokinetic study: SL, NC. Contributed with included patients: LS, FA-L, MM, SH, EE. All authors read and approved the final manuscript.

\section{Competing interests}

The authors declare that they have no competing interests.

\section{Consent for publication}

Participant's consent was also obtained for the purposes of publishing the results from this study. All the authors consented to the study results to be published in the form presented in the final version of this manuscript.

\section{Ethics approval and consent to participate}

All participants were informed about the study's purpose and procedures. Written informed consent was obtained from all patients or their legal representatives. The ethics committee of the hospital (Comitè Ètic de Investigació Clínica del Parc de Salut MAR) approved the study.

\section{Author details}

${ }^{1}$ Infectious Disease Service, Hospital del Mar, Barcelona, Spain. ${ }^{2}$ Pharmacy Service, Hospital del Mar, Barcelona, Spain. ${ }^{3}$ Institut Hospital del Mar d'Investigacions Mèdiques (IMIM), Barcelona, Spain. ${ }^{4}$ Laboratori de Referència de Catalunya, Prat de Llobregat, Spain. ${ }^{5}$ InfectiousDiseaseUnit, Department of Internal Medicine, Hospital de la Santa Creu i Sant Pau, Barcelona, Spain. ${ }^{6}$ Institut d'Investigació Biomèdica Sant Pau, Barcelona, Spain. ${ }^{7}$ Spanish Network for Research in Infectious Diseases (REIPI RD12/0015), Instituto de Salud Carlos III, Madrid, Spain. ${ }^{8}$ Intensive Care Unit, Hospital del Mar, Barcelona, Spain. ${ }^{9} \mathrm{CIBERES}$, Madrid, Spain. ${ }^{10}$ Universitat Autònoma de Barcelona, Barcelona, Spain. ${ }^{11}$ CEXS-Universitat Pompeu Fabra, Barcelona, Spain.

\section{Received: 20 April 2016 Accepted: 14 December 2016}

\section{Published online: 05 January 2017}

\section{References}

1. Shorr AF. Review of studies of the impact on Gram-negative bacterial resistance on outcomes in the intensive care unit. Crit Care Med. 2009;37: 1463-9. Department of Pulmonary and Critical Care Medicine, Washington Hospital Center, Washington DC, USA. afshorr@mail.dnamail.com.

2. Boucher HW, Talbot GH, Bradley JS, Edwards JE, Gilbert D, Rice LB, et al. Bad bugs, no drugs: no ESKAPE! An update from the Infectious Diseases Society of America. Clin Infect Dis. 2009;48:1-12. Division of Geographic Medicine and Infectious Diseases, Tufts University and Tufts Medical Center, Boston, Massachusetts 02111, USA. hboucher@tuftsmedicalcenter.org.

3. Payne DJ, Gwynn MN, Holmes DJ, Pompliano DL. Drugs for bad bugs: confronting the challenges of antibacterial discovery. Nat Rev Discov. 2007;6:29-40. Infectious Diseases Centre of Excellence for Drug Discovery, GlaxoSmithKline, 1250 South Collegeville Road, Collegeville, Pennsylvania 19426, USA.

4. Bergen PJ, Landersdorfer CB, Zhang J, Zhao M, Lee HJ, Nation RL, et al. Pharmacokinetics and pharmacodynamics of 'old' polymyxins: what is new? Diagn Microbiol Infect Dis. 2012;74:213-23. Drug Delivery, Disposition and Dynamics, Monash Institute of Pharmaceutical Sciences, Monash University, Parkville, Victoria 3052, Australia.: Elsevier Inc.

5. Michalopoulos AS, Karatza DC, Gregorakos L. Pharmacokinetic evaluation of colistin sodium. Expert Opin Drug Metab Toxicol. 2011;7:245-55. Intensive Care Unit, Henry Dunant Hospital, 107 Mesogeion Ave, 11528, Athens, Greece. amichalopoulos@hol.gr.

6. Garonzik SM, Li J, Thamlikitkul V, Paterson DL, Shoham S, Jacob J, et al. Population pharmacokinetics of colistin methanesulfonate and formed colistin in critically ill patients from a multicenter study provide dosing suggestions for various categories of patients. Antimicrob Agents Chemother. 2011;55:3284-94. School of Pharmacy and Pharmaceutical Sciences, University at Buffalo, SUNY, Buffalo, New York, USA.

7. Yahav D, Farbman L, Leibovici L, Paul M. Colistin: new lessons on an old antibiotic. Clin Microbiol Infect. 2012;18:18-29. Internal Medicine E, Rabin Medical Centre, Beilinson Hospital, Petah Tikva, Israel. dafna.yahav@gmail. com: European Society of Clinical Microbiology and Infectious Diseases. Available from: http://www.ncbi.nlm.nih.gov/pubmed/22168320.

8. Falagas ME, Rafailidis PI, loannidou E, Alexiou VG, Matthaiou DK, Karageorgopoulos DE, et al. Colistin therapy for microbiologically documented multidrug-resistant Gram-negative bacterial infections: a retrospective cohort study of 258 patients. Int J Antimicrob Agents. 2010;35: 194-9. Alfa Institute of Biomedical Sciences (AIBS), 9 Neapoleos Street, 15 123 Marousi, Athens, Greece.m.falagas@aibs.gr: Elsevier B.V. and the International Society of Chemotherapy. All rights reserved.

9. Montero M, Horcajada JP, Sorli L, Alvarez-Lerma F, Grau S, Riu M, et al. Effectiveness and safety of colistin for the treatment of multidrug-resistant Pseudomonas aeruginosa infections. Infection. 2009;37:461-5. Dept. of Internal Medicine and Infectious Diseases, Hospital del Mar, Autonomous University of Barcelona, Barcelona, Spain. 95422@imas.imim.es.

10. Dalfino L, Puntillo F, Mosca A, Monno R, Spada ML, Coppolecchia S, et al. High-dose, extended-interval colistin administration in critically ill patients: is this the right dosing strategy? A preliminary study. Clin Infect Dis. 2012:54:1720-6. Anesthesia and Intensive Care Unit, Department of Emergeny and Organ Transplantion.

11. Paul M, Bishara J, Levcovich A, Chowers M, Goldberg E, Singer $P$, et al. Effectiveness and safety of colistin: prospective comparative cohort study. J Antimicrob Chemother. 2010;65:1019-27. Unit of Infectious Diseases, Rabin Medical Center, Beilinson Hospital, Petah Tikva, Israel. paulm@post.tau.ac.il. Available from: http://www.ncbi.nlm.nih.gov/ pubmed/20299494.

12. Ortwine JK, Kaye KS, Li J, Pogue JM. Colistin: understanding and applying recent pharmacokinetic advances. Pharmacotherapy. 2015;35:11-6. Available from: http://www.ncbi.nlm.nih.gov/pubmed/25187500.

13. Foundation NK. K/DOQI clinical practice guidelines for chronic kidney disease: evaluation, classification, and stratification. Am J Kidney Dis. 2002;39:S1-266.

14. Charlson M, Szatrowski TP, Peterson J, Gold J. Validation of a combined comorbidity index. J Clin Epidemiol. 1994;47:1245-51. Department of Medicine, Cornell Arthritis and Musculoskeletal Disease Center, Hospital for Special Surgery, New York, NY, USA.

15. Knaus WA, Draper EA, Wagner DP, Zimmerman JE. APACHE II: a severity of disease classification system. Crit Care Med. 1985;13:818-29.

16. Horan TC, Andrus M, Dudeck MA. CDC/NHSN surveillance definition of health care-associated infection and criteria for specific types of infections in the acute care setting. Am J Infect Control. 2008;36:309-32. National Healthcare Safety Network, Division of Healthcare Quality Promotion, Centers for Disease Control and Prevention, Atlanta, GA 30333, USA. thoran@cdc.gov. Available from: http://www.ncbi.n/m.nih.gov/pubmed/ 18538699

17. American College of Chest Physicians/Society of Critical Care Medicine Consensus Conference: definitions for sepsis and organ failure and 
guidelines for the use of innovative therapies in sepsis. Crit. Care Med. 1992;20:864-74.

18. Magiorakos A-P, Srinivasan A, Carey RB, Carmeli Y, Falagas ME, Giske CG, et al. Multidrug-resistant, extensively drug-resistant and pandrug-resistant bacteria: an international expert proposal for interim standard definitions for acquired resistance. Clin Microbiol Infect. 2012;18:268-81. Available from: http://www.ncbi.nlm.nih.gov/pubmed/21793988.

19. Li J, Milne RW, Nation RL, Turnidge JD, Coulthard K, Johnson DW. A simple method for the assay of colistin in human plasma, using pre-column derivatization with 9-fluorenylmethyl chloroformate in solid-phase extraction cartridges and reversed-phase high-performance liquid chromatography J Chromatogr Biomed Sci Appl. 2001;761:167-75. Centre for Pharmaceutical Research, University of South Australia, Adelaide Australia.

20. Sorlí L, Luque S, Grau S, Berenguer N, Segura C, Montero MM, et al. Trough colistin plasma level is an independent risk factor for nephrotoxicity: a prospective observational cohort study. BMC Infect Dis. 2013;13:380. Available from: http://www.pubmedcentral.nih.gov/articlerender.fcgi?artid= 3765824\&tool=pmcentrez\&rendertype $=$ abstract.

21. Couet W, Gregoire N, Marchand S, Mimoz O. Colistin pharmacokinetics: the fog is lifting. Clin Microbiol Infect. 2012;18:30-9. Inserm ERI-23, CHU, Service de Toxicologie et Pharmacocinetique, UFR Medecine-Pharmacie, Universite de Poitiers Intensive Care Unit, University Hospital of Poitiers, Poitiers, France.: European Society of Clinical Microbiology and Infectious Diseases.

22. Bellomo R, Ronco C, Kellum JA, Mehta RL, Palevsky P, ADQI. Acute renal failure - definition, outcome measures, animal models, fluid therapy and information technology needs: the Second International Consensus Conference of the Acute Dialysis Quality Initiative (ADQI) Group. Crit Care. 2004;8:R204-12. Department of Intensive Care and Medicine, Austin Health, Melbourne, Australia. rinaldo.bellomo@austin.org.au.

23. Levin AS, Barone AA, Penco J, Santos MV, Marinho IS, Arruda EA, et al. Intravenous colistin as therapy for nosocomial infections caused by multidrug-resistant Pseudomonas aeruginosa and Acinetobacter baumannii. Clin Infect Dis. 1999;28:1008-11. Hospital Infection Control Department, Hospital das Clinicas, Faculdade de Medicina, University of Sao Paulo, SP Brazil. nivel@usp.br.

24. Cheng $C Y$, Sheng WH, Wang JT, Chen YC, Chang SC. Safety and efficacy of intravenous colistin (colistin methanesulphonate) for severe multidrugresistant Gram-negative bacterial infections. Int J Antimicrob Agents. 2010; 35:297-300. Department of Internal Medicine, National Taiwan University Hospital, Taipei, Taiwan.: Elsevier B.V. and the International Society of Chemotherapy. All rights reserved.

25. Markou N, Apostolakos H, Koumoudiou C, Athanasiou M, Koutsoukou A, Alamanos I, et al. Intravenous colistin in the treatment of sepsis from multiresistant Gram-negative bacilli in critically ill patients. Crit Care. 2003;7: R78-83. Intensive Care Unit B, Athens Trauma Hospital KAT, Athens, Greece. nikolaos_markou@hotmail.com.

26. Plachouras D, Karvanen M, Friberg LE, Papadomichelakis E, Antoniadou A, Tsangaris I, et al. Population pharmacokinetic analysis of colistin methanesulfonate and colistin after intravenous administration in critically ill patients with infections caused by gram-negative bacteria. Antimicrob Agents Chemother. 2009:53:3430-6. 4th Department of Internal Medicine, Attikon University General Hospital, Haidari 12462, Greece. dplach@med.uoa.gr

27. Markou N, Markantonis SL, Dimitrakis E, Panidis D, Boutzouka E, Karatzas S, et al. Colistin serum concentrations after intravenous administration in critically ill patients with serious multidrug-resistant, gram-negative bacilli infections: A prospective, open-label, uncontrolled study. Clin Ther. 2008:30:143-51. Available from: http://www.sciencedirect.com/science/ article/pii/S0149291808000635.

28. Yilmaz GR, Bastug AT, But A, Yildiz S, Yetkin MA, Kanyilmaz D, et al. Clinical and microbiological efficacy and toxicity of colistin in patients infected with multidrug-resistant gram-negative pathogens. J Infect Chemother. 2013;19:57-62. Infectious Diseases and Clinical Microbiology Department, Ankara Numune Education and Research Hospital, Ankara, Turkey.ruhsar6@yahoo.com.

29. Zaidi ST, Al Omran S, Al Aithan AS, Al Sultan M. Efficacy and safety of lowdose colistin in the treatment for infections caused by multidrug-resistant gram-negative bacteria. J Clin Pharm Ther. 2014;39(3):272-6.

30. Khawcharoenporn T, Pruetpongpun N, Tiamsak P, Rutchanawech S, Mundy LM, Apisarnthanarak A. Colistin-based treatment for extensively drug-resistant Acinetobacter baumannii pneumonia. Int J Antimicrob Agents. 2014;43:378-82.
31. Peña C, Gómez-Zorrilla S, Oriol I, Tubau F, Dominguez MA, Pujol M, et al. Impact of multidrug resistance on Pseudomonas aeruginosa ventilatorassociated pneumonia outcome: predictors of early and crude mortality. Eur J Clin Microbiol Infect Dis. 2013;32:413-20. Available from: http://www. ncbi.nlm.nih.gov/pubmed/23344827.

32. Kwon KH, Oh JY, Yoon Y-S, Jeong Y-J, Kim KS, Shin SJ, et al. Colistin treatment in carbapenem-resistant Acinetobacter baumannii pneumonia patients: Incidence of nephrotoxicity and outcomes. Int J Antimicrob Agents. 2015;45(6):605-9. Available from: http://www.ncbi.nlm.nih.gov/ pubmed/25772644.

33. Rigatto MH, Oliveira MS, Perdigão-Neto LV, Levin AS, Carrilho CM, Tanita MT, et al. Antimicrob Agents Chemother. 2016;60:2443-9. Available from: http://www.ncbi.nlm.nih.gov/pubmed/26856846.

34. Gurjar M. Colistin for lung infection: an update. J intensive care. 2015;3:3. Available from: http://www.pubmedcentral.nih.gov/articlerender.fcgi?artid= 4336271\&tool=pmcentrez\&rendertype=abstract.

\section{Submit your next manuscript to BioMed Central and we will help you at every step:}

- We accept pre-submission inquiries

- Our selector tool helps you to find the most relevant journal

- We provide round the clock customer support

- Convenient online submission

- Thorough peer review

- Inclusion in PubMed and all major indexing services

- Maximum visibility for your research

Submit your manuscript at www.biomedcentral.com/submit
C Biomed Central 